\title{
COMPARATIVO DE EMPADRE CONTROLADO EN CUYES CON MACHOS QUE DESCANSAN EN SUS POZAS DE EMPADRE $V S$. DESCANSO EN POZAS INDIVIDUALES, CON Y SIN EFECTO MACHO
}

\author{
Luis H. Aliaga Rodríguez* \\ Universidad Católica Sedes Sapientiae y Agropecuaria Inka Cuy S. A. C. \\ laliaga@ucss.edu.pe \\ Luis A. Aliaga Rota** \\ Universidad Católica Sedes Sapientiae y Agropecuaria Inka Cuy S. A. C. \\ laliagarota1@yahoo.es \\ Jaime Rodriguez Vilcapoma ${ }^{* *}$ \\ Agropecuaria Inka Cuy S. A. C. \\ jaime_rodriguez12@hotmail.com
}

Fecha de recepción: agosto de 2015

Fecha de aceptación: octubre de 2015

Resumen: El presente trabajo se desarrolló en la empresa Agropecuaria Inka Cuy S. A. C., ubicada en el kilómetro 50 de la carretera Lima Canta, distrito de Santa Rosa de Quives, provincia de Canta, Región Lima, a una altitud de 1,000 m.s.n.m., durante enero del 2014 a diciembre del 2014.

*Vicerrector académico de la Universidad Católica Sedes Sapientiae (UCSS).

** Docente ordinario de la Facultad de Ingeniería Agraria de la UCSS.

${ }^{\cdots}$ Responsable de la Sección Cuyes en la empresa Agropecuaria Inka Cuy S. A. C. 
El objetivo fue evaluar la respuesta productiva en el primer parto de madres que, durante su fase reproductiva, se empadraron con machos que descansaron en sus propias pozas de empadre (T1) vs. madres empadradas con machos que descansaron en pozas individuales (maternidades) (T2) vs. madres empadradas con machos que descansaron en pozas individuales (maternidades) en contacto indirecto con las hembras para lograr el efecto macho (T3). Igualmente, las hembras de los tres tratamientos parieron en maternidades. A su vez, se evaluaron dos empadres con hembras vírgenes (2 lotes) y se registró en la parición los siguientes parámetros reproductivos: (a) tamaño de camada, (b) mortalidad de crías desde el nacimiento hasta el destete, (c) número de crías destetadas por cada hembra parida, y (d) número y peso de crías destetadas por cada hembra empadrada.

Asimismo, se realizó en la parte final un análisis económico de los resultados. Los resultados obtenidos demuestran un tamaño de camada para el tratamiento T1 de 2.97; T2 de 3.12 y T3 de 3.29 crías que, al análisis de variancia, resultaron no ser significativas. Juntamente, el número de crías destetadas por cada hembra empadrada para el T1 fue de 1.84 crías vs. 2.18 para el T2 y 2.21 para el T3. Estas diferencias, al análisis de variancia, resultaron no ser significativas. Después, se concluye precisando que el descanso del macho en una poza individual, en contacto indirecto con las hembras por 15 días denominado efecto macho (T3) vs. el descanso del macho en su propia poza de empadre (T1), utilizado por la empresa Inka Cuy S.A.C., permite un incremento del tamaño de camada; del número y peso de crías destetadas por hembra empadrada; y del valor bruto de la producción en soles, cuya diferencia a favor del T3 con efecto macho vs. el T1 sin efecto macho es de 3.30 soles de crías destetadas por cada hembra empadrada. Cabe afirmar que dicha cantidad, para una explotación de cuyes donde normalmente se empadran miles de animales, resulta interesante. 
Palabras Clave: Empadre, tratamientos, partos, maternidades, destete, crías.

\section{MATING COMPARATIVE CONTROLLED IN GUINEA PIGS WITH MALES WHO REST IN HIS PUDDLES'S MATING VS. REST IN INDIVIDUAL PUDDLES, WITH AND WITHOUT MALE EFFECT}

ABstract: The present work developed in the Agropecuaria Inka Cuy S.A.C., located in the kilometre 50 of the road Lima Canta, district of Quives's Santa Rosa, province of Canta, Region Lima, to an altitude of 1,000 m.s.n.m., during January, 2014 to December, 2014. The aim was to evaluate the productive response in the mothers' first childbirth that, during his reproductive phase, they had mated with males that mothers rested in his own puddles of mating (T1) vs. mated mothers with males that mothers rested in individual puddles (maternities) (T2) vs. mated mothers with males who rested in individual puddles (maternities) in indirect contact with the females to achieve the male effect (T3). Equally, the females of three treatments gave birth in maternities. In turn, there evaluated two matings with females virgins (2 lots) and one registered in the parturition the following reproductive parameters: (a) size of litter, (b) babies' mortality from the birth up to the weaning, (c) number of babies weaned by every given birth female, and (d) number and babies' weight weaned by every mated female.

Likewise, an economic analysis of the results was realized in the final part. The obtained results demonstrate a size of litter for the treatment 
$\mathrm{T} 1$ of 2.97 ; $\mathrm{T} 2$ of 3.12 and T3 of 3.29 babies who, to the variance analysis, proved not to be significant. Unitedly, the number of babies weaned by every mated female for the T1 belonged 1.84 babies vs. 2.18 for T2 and 2.21 for the T3. These differences, to the variance analysis, proved not to be significant. Later, it ends up by specifying that the rest of the male in an individual puddle, in indirect contact with the females for 15 days named male effect (T3) vs. the rest of the male in his own puddle of mating (T1), used by the company Inka Cuy S.A.C., it allows an increase of the size of litter; of the number and babies' weight weaned by mated female; and of the brute value of the production in soles, which difference in favour of the T3 with male effect vs. the T1 without effect male is 3.30 babies' soles weaned by every mated famale. It is necessary to affirm that the above mentioned quantity, for an exploitation of guinea pigs where normally to mate thousands of animals, it turns out to be interesting.

KEYwORDs: Mating, treatments, childbirths, maternities, weaning, babies.

\section{Introducción}

1 estudio presentado se desarrolló en la empresa Agropecuaria Inka
Cuy S.A.C., ubicada en el kilómetro 50 de la carretera Lima Canta,
distrito de Santa Rosa de Quives, provincia de Canta, Región Lima, a una altitud de 1,000 m.s.n.m. Precisamente, el proceso de evaluación de cuyes nenonatos se realizó durante febrero del 2014 hasta diciembre del mismo año. Se evaluó la respuesta productiva en el primer parto de madres que, durante su proceso de la reproducción, fueron sometidas a un empadre controlado con machos que descansaron, antes del empadre, en sus propias pozas de empadre; es decir, prolongando el periodo de empadre 


\section{COMPARATIVO DE EMPADRE CONTROLADO EN CUYES}

de 35 a 55 días, (T1), vs. madres que durante su empadre de 35 días, se empadronaron con machos que descansaron 15 días adicionales en pozas individuales (maternidades) (T2), vs. madres que, durante su empadre de 35 días, se empadraron con machos que descansaron 15 días adicionales en pozas individuales (maternidades) en contacto indirecto con las hembras a empadrarse para provocar el efecto macho en ellas (T3).

Conjuntamente, las hembras de los tres tratamientos fueron sometidas a un flushing proteico y energético a través de un concentrado con $21 \%$ de proteína y 3,000 kilo calorías de energía durante 15 días antes del inicio del empadre. El ensayo tuvo dos repeticiones (2 lotes), en otras palabras, dos empadres en dos fechas diferentes. De igual modo, se evaluaron en los dos partos (2 lotes) los siguientes parámetros productivos: (a) peso de madres al inicio del empadre, (b) tamaño de camada, (c) mortalidad de crías desde el nacimiento hasta el destete y número y (d) peso de crías destetadas por cada hembra empadrada. Finalmente, se hizo un análisis económico de los resultados.

\section{Materiales y Métodos}

El presente trabajo, que abarcó un año entre enero del 2014 hasta diciembre del 2014, se llevó a cabo en la empresa Agropecuaria Inka Cuy S.A.C. Esta se ubica en el kilómetro 50 de la carretera Lima Canta, distrito de Santa Rosa de Quives, provincia de Canta, Región Lima. Asimismo, se encuentra a una altitud de 1,000 m.s.n.m.

De igual modo, el objetivo fue evaluar la respuesta productiva en el primer parto de madres. Estas, durante el proceso de la reproducción, fueron sometidas a empadre controlado con machos que descansaron, antes del empadre, en sus propias pozas de empadre. Es decir, se prolongó 15 
días el período de empadre de 35 a 55 días (T1), vs. madres que durante su empadre controlado de 35 días, fueron empadradas con machos que descansan durante 15 días en pozas individuales (maternidades) (T2), vs. madres que, durante su empadre controlado de 35 días, fueron empadradas con machos que descansaron durante 15 días en pozas individuales (maternidades) en contacto indirecto con las hembras a empadrarse para provocar el efecto macho en la hembras (T3).

Las hembras de los tres tratamientos fueron sometidas a un flushing proteico y energético a través de un concentrado con $21 \%$ de proteína y 3,000 kilo calorías de energía. El tiempo utilizado fue durante 15 días antes del inicio del empadre.

Por otro lado, la población utilizada constó de 77 hembras de dos meses de edad con pesos superiores a $600 \mathrm{~g}$ y 7 machos de 3 meses de edad con pesos superiores a $1,200 \mathrm{~g}$. En los tres tratamientos, hembras y machos fueron sometidos a las mismas condiciones de alimentación y manejo para evitar la influencia de factores extraños a los tratamientos en estudio. Se ubicó a los animales en un galpón con 7 pozas de empadre de madera de 1.50 × $1.50 \mathrm{~m}$, con piso de malla de alambre, levantadas con patas de madera (2 para T1; 2 para T2 y 3 para T3) y 77 maternidades de la misma estructura de $0.50 \mathrm{~m}$ x $0.30 \mathrm{~m}$ para la parición.

Sobre los tratamientos, se compararon dos tratamientos de empadre con machos que descansaron en sus propias pozas de empadre (T1) vs. empadre con machos que descansan en pozas individuales (maternidades) (T2). vs. empadre con machos que descansaron en pozas individuales (maternidades) en contacto indirecto con las hembras para lograr el efecto macho (T3). Dichos tratamientos serán especificados más adelante. En cuanto al diseño experimental, se utilizó el denominado "Arreglo Factorial de un Completamente Randomizado de 3 tratamientos por 2 lotes". 


\section{Tratamientos y Variables de Estudio}

Los tratamientos estudiados fueron tres. Estos se denominaron T1, T2 y T3. A continuación, se explicará cada uno de ellos:

- $\quad \mathrm{T} 1=$ Empadre controlado con machos que descansan antes del empadre, en sus propias pozas de empadre, o sea, prolongando 15 días el período de empadre de 35 a 55 días.

- $\quad \mathrm{T} 2=$ Empadre controlado de 35 días, con machos que descansan durante 15 días adicionales en pozas individuales (maternidades).

- $\quad$ T3= Empadre controlado de 35 días, con machos que descansan durante 15 días adicionales en pozas individuales (maternidades) en contacto indirecto con las hembras a empadrarse para provocar el efecto macho en la hembras.

Cabe mencionar que las hembras de los tres tratamientos fueron sometidas a un flushing proteico y energético a través de un concentrado con 21\% de proteína y 3,000 kilo calorías de energía, durante 15 días antes del inicio del empadre. Simultáneamente, se evaluaron (a) el peso de las hembras al empadre, (b) el tamaño de camada, (c) el número de crías destetadas por cada hembra parida y empadrada, (d) el peso total de camada destetada por cada hembra empadrada y, finalmente, (e) un análisis económico sobre los resultados. Dichos datos también serán detallados más adelante en tablas. 


\section{Resultados y Discusión}

Luego de proponer las premisas en torno a los tres tratamientos evaluados, se procederá a representar los resultados de su evaluación. En la Tabla 1, se explica el registro de pesos de machos durante el proceso de empadre de lotes de hembras. En esta se detallan las fechas de inicio y fin del empadre del primer y segundo lote, así como el peso del empadre del primer lote y otros detalles referidos al incremento del peso de los machos. 


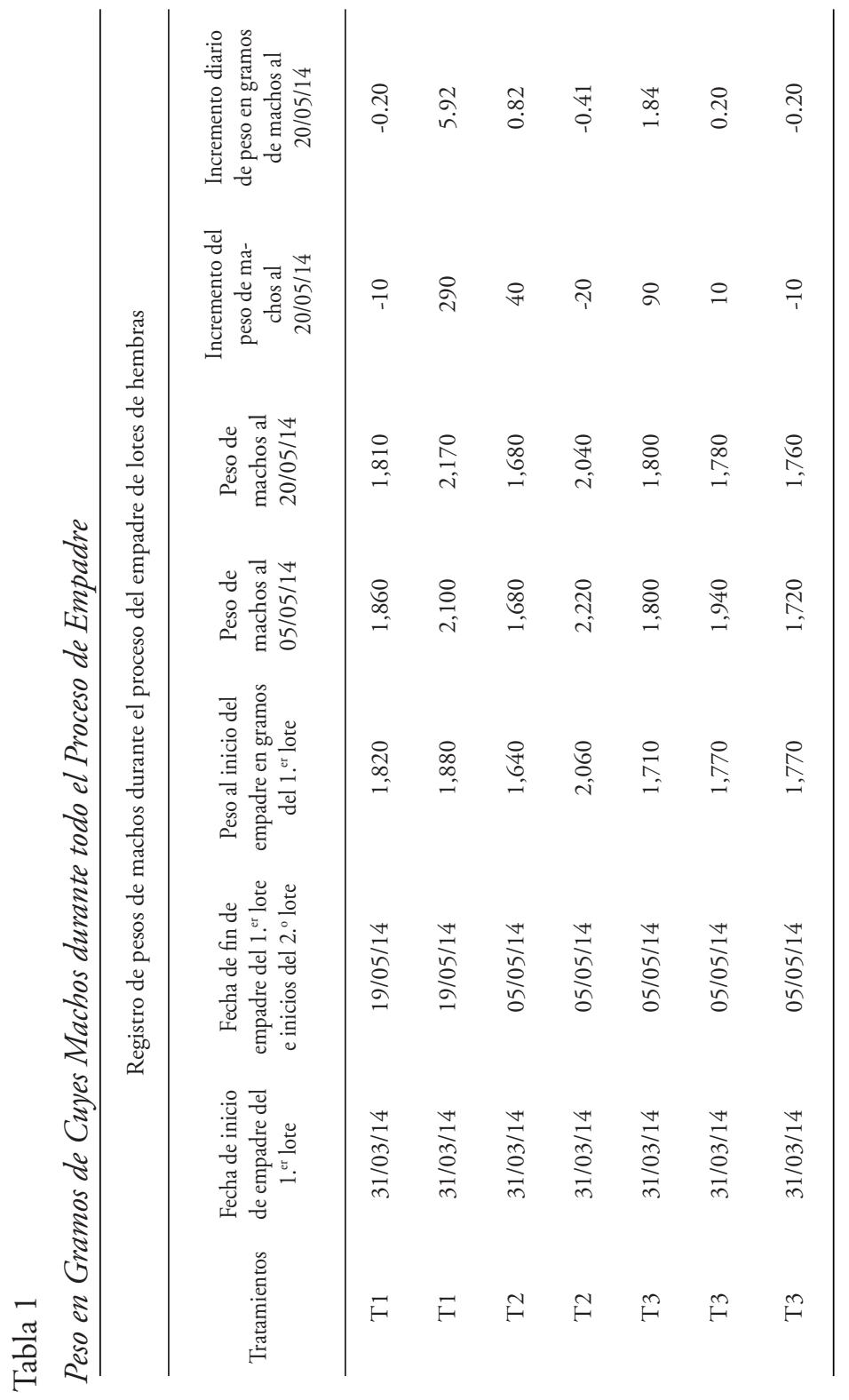


En la Tabla 2, se observará el peso en gramos de cuyes y hembras al inicio del empadre por lotes y tratamientos. También se consignará el número de hembras, el peso promedio de tratamientos y el peso promedio de lotes. Asimismo, se presentará el respectivo análisis de la información obtenida.

\section{Tabla 2}

Peso en Gramos de Cuyes Hembras al Inicio del Empadre por Lotes y Tratamientos

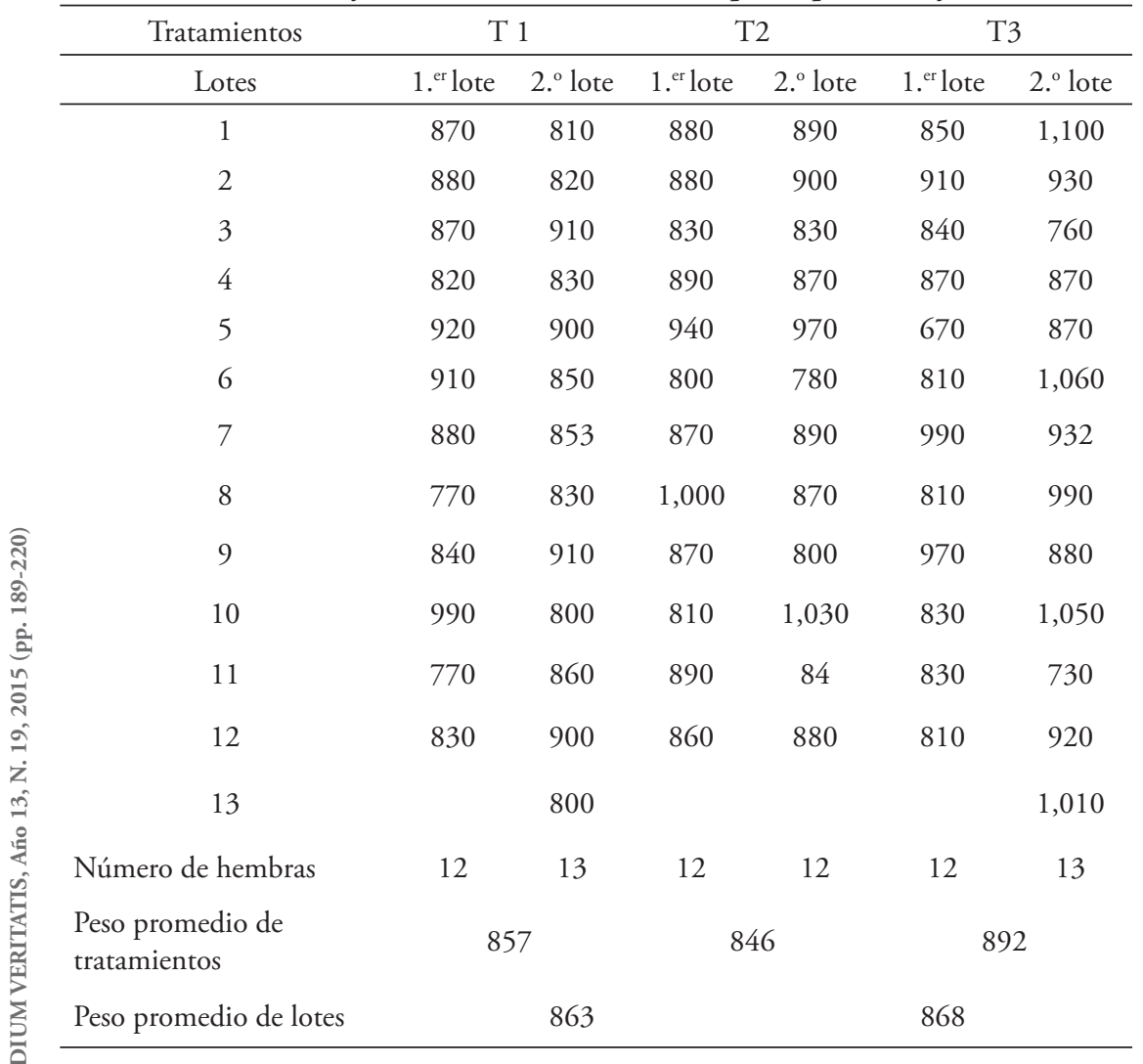




\section{COMPARATIVO DE EMPADRE CONTROLADO EN CUYES}

En la Tabla 3 se ubicará el análisis de variancia del peso en gramos de cuyes hembras al inicio del empadre por lotes y tratamientos. A partir de este análisis, podrá inferirse que los pesos al inicio del empadre de los tres tratamientos (ver Tabla 2) son semejantes con un 99\% de seguridad. Se descartará, por lo tanto, la posible influencia de este parámetro en los resultados del ensayo. A continuación, se detallará la información.

Tabla 3

Análisis de Variancia del Peso en Gramos de Cuyes Hembras al Inicio del Empadre por Lotes y Tratamientos

\begin{tabular}{lcccccccc}
\hline \multicolumn{1}{c}{ F. V } & SC & GL & CM & FC & Ft, 05 & SIG & Ft, 01 & SIG \\
\hline Entre combinaciones & 89094.86 & 5 & 17818.97 & 1.282 & 2.370 & No & 3.340 & No \\
Entre tratamientos & 27056.58 & 2 & 13528.34 & 0.973 & 3.150 & No & 4.980 & No \\
Entre lotes & 463.21 & 1 & 463.21 & 0.033 & 4.000 & No & 7.090 & No \\
Entre interacción T x L & 61574.97 & 2 & 30787.48 & 2.215 & 3.150 & No & 4.980 & No \\
Error Experimental & 9590814.18 & 69 & 13899.73 & & & & & \\
Total & 1048176.04 & 74 & & & & & & \\
\multicolumn{1}{c}{$\mathrm{CV}=$} & 13.62 & & & & & & & \\
\hline
\end{tabular}

En la Tabla 4, se apreciará al tamaño, fertilidad y mortalidad de hembras por lotes y tratamientos. Se observarán, además, el tamaño de camada correspondiente a 2.97 crías para las hembras empadradas con machos que descansaron en sus propias pozas de empadre (T1) vs. el resultado de 3.12 crías para las hembras empadradas con machos que descansaron en pozas individuales (maternidades) (T2) y 3.29 crías para las hembras empadradas con machos que descansaron e hicieron el efecto macho en pozas individuales (maternidades) (T3). 


\section{LUIS H. ALIAGA RODRÍGUEZ}

\section{Tabla 4}

\section{Tamaño de Camada, Fertilidad y Mortalidad de Hembras por Lotes y Tratamientos}

\begin{tabular}{|c|c|c|c|c|c|c|}
\hline Tratamientos & \multicolumn{2}{|c|}{ T1 } & \multicolumn{2}{|c|}{ T2 } & \multicolumn{2}{|c|}{ T3 } \\
\hline Lotes & 1. ${ }^{\text {er }}$ lote & 2. ${ }^{\circ}$ lote & 1. ${ }^{\text {er }}$ lote & $2 .^{\circ}$ lote & 1. ${ }^{\text {er }}$ lote & 2. lote \\
\hline 1 & 3 & 4 & 3 & 2 & 1 & 3 \\
\hline 2 & 3 & Vacía & 3 & 3 & 3 & 3 \\
\hline 3 & 3 & Vacía & 3 & 2 & 4 & Vacía \\
\hline 4 & 4 & Vacía & 4 & 3 & 2 & 4 \\
\hline 5 & Vacía & Vacía & 3 & 3 & 2 & 3 \\
\hline 6 & 3 & 3 & 3 & 3 & 3 & 3 \\
\hline 7 & Vacía & 3 & 4 & 2 & 4 & Vacía \\
\hline 8 & 3 & Vacía & 2 & 3 & 4 & 3 \\
\hline 9 & 3 & 3 & 4 & 2 & Vacía & Vacía \\
\hline 10 & 3 & 3 & 3 & 4 & Vacía & 3 \\
\hline 11 & 3 & 3 & 5 & 4 & 3 & Murió \\
\hline 12 & Vacía & & 4 & & 3 & \\
\hline 13 & 3 & & 3 & & 2 & \\
\hline 14 & 3 & 3 & Vacía & Vacía & 1 & Vacía \\
\hline 15 & Vacía & 2 & 4 & Vacía & 4 & Murió \\
\hline 16 & 3 & 3 & Murió & Vacía & 3 & Vacía \\
\hline 17 & 3 & 2 & 4 & 2 & 3 & 4 \\
\hline 18 & 3 & 2 & Vacía & Vacía & 4 & 4 \\
\hline 19 & Vacía & 2 & 2 & 4 & 2 & 3 \\
\hline 20 & 3 & Vacía & 3 & 3 & 4 & Vacía \\
\hline 21 & Vacía & 3 & Vacía & Vacía & 3 & Vacía \\
\hline 22 & Murió & 3 & 2 & Vacía & 4 & 3 \\
\hline 23 & & 4 & & 3 & & 4 \\
\hline 24 & & & & 4 & 3 & Murió \\
\hline 25 & & & & & 4 & \\
\hline 26 & & & & & 3 & \\
\hline 27 & & & & & 4 & 3 \\
\hline 28 & & & & & 4 & Vacía \\
\hline 29 & & & & & 5 & Murió \\
\hline 30 & & & & & 4 & 3 \\
\hline 31 & & & & & 4 & 4 \\
\hline 32 & & & & & Vacía & 4 \\
\hline 33 & & & & & Murió & 4 \\
\hline 34 & & & & & & Vacía \\
\hline 35 & & & & & & Murió \\
\hline 36 & & & & & & Perdida \\
\hline 37 & & & & & & Perdida \\
\hline 38 & & & & & & \\
\hline 39 & & & & & & \\
\hline Suma de combinaciones & $\Phi$ & $\Phi$ & $\Phi$ & $\Phi$ & $\Phi$ & $\Phi$ \\
\hline N. Rep en combinaciones & 15 & 15 & 18 & 16 & 28 & 17 \\
\hline Promedio de combinaciones & 3.07 & 2.87 & 3.28 & 2.94 & 3.21 & 3.41 \\
\hline Promedio de tratamientos & & $7^{2.07}$ & & & & $29^{5.41}$ \\
\hline Promedio de lotes de hembras & & 3.20 & & & 3.08 & \\
\hline Porcentaje de vacías + muertos & & & & & & \\
\hline oor combinaciones & $31.82 \%$ & $27.27 \%$ & $13.64 \%$ & $27.27 \%$ & $12.12 \%$ & $27.27 \%$ \\
\hline
\end{tabular}




\section{COMPARATIVO DE EMPADRE CONTROLADO EN CUYES}

En la Tabla 5, se procederá al análisis de variancia del tamaño de camada por cada hembra parida, tanto por lotes como por tratamientos. Vistos los resultados del análisis, las variables son semejantes para $(\mathrm{p}<0.01)$. Sin embargo, la diferencia de crías entre el T1, que corresponde al sistema de empadre actual que emplea la Agropecuaria Inka Cuy S.A.C., y el T3, de 0.32 crías a favor de este último tratamiento, resulta ser significativa, pues se tendrá también mayor número de crías destetadas como se verá más adelante.

Tabla 5

Análisis de Variancia del Tamaño de Camada por cada Hembra Parida por Lotes y Tratamientos

\begin{tabular}{|c|c|c|c|c|c|c|c|c|}
\hline F.V. & SC & GL & $\mathrm{CM}$ & FC & $\mathrm{Ft}, 05$ & SIG & $\mathrm{Ft}, 01$ & SIG \\
\hline Entre combinaciones & 3.60 & 5 & 0.721 & 1.279 & 2.290 & No & 3.170 & No \\
\hline Entre tratamientos & 1.91 & 2 & 0.955 & 1.695 & 3.070 & No & 4.790 & No \\
\hline Entre lotes & 0.35 & 1 & 0.345 & 0.613 & 3.920 & No & 6.850 & No \\
\hline Entre interacción $\mathrm{T}$ x L & 1.35 & 2 & 0.674 & 1.196 & 3.070 & No & 4.790 & No \\
\hline Error Experimental & 58.05 & 103 & 0.564 & & & & & \\
\hline Total & 61.65 & 108 & & & & & & \\
\hline $\mathrm{CV}=$ & 23.86 & & & & & & & \\
\hline
\end{tabular}

Seguidamente, en la Tabla 6, se especificará el número de crías destetadas por cada hembra parida por lotes y tratamientos. De acuerdo con ello, figurarán 2.79 crías para las hembras empadradas con machos que descansaron en sus propias pozas de empadre (T1) vs. 2.82 crías para las hembras empadradas con machos que descansaron en pozas individuales (maternidades) (T2) y 3.17 crías para las hembras empadradas con machos que descansaron e hicieron el efecto macho en pozas individuales (maternidades) (T3). A continuación, se desarrollará la información mencionada. 


\section{LUIS H. ALIAGA RODRÍGUEZ}

\section{Tabla 6}

Número de Crías Destetadas por cada Hembra Parida por Lotes y Tratamientos

\begin{tabular}{|c|c|c|c|c|c|c|}
\hline Tratamientos & \multicolumn{2}{|c|}{$\mathrm{T} 1$} & \multicolumn{2}{|c|}{ T2 } & \multicolumn{2}{|c|}{ T3 } \\
\hline Lotes & 1. ${ }^{\text {er }}$ lote & $2 .^{\circ}$ lote & $1 .^{\text {er }}$ lote & $2 .^{\circ}$ lote & $1 .^{\text {er }}$ lote & $2 .^{\circ}$ lote \\
\hline 1 & 3 & 4 & 3 & 2 & 1 & 3 \\
\hline 2 & 3 & & 3 & 2 & 3 & 4 \\
\hline 3 & 3 & & 2 & 2 & 4 & \\
\hline 4 & 4 & & 3 & 3 & 2 & 4 \\
\hline 5 & & & 3 & 2 & 2 & 3 \\
\hline 6 & 3 & 3 & 3 & 3 & 3 & 3 \\
\hline 7 & & 3 & 4 & 2 & 4 & \\
\hline 8 & 3 & & 2 & 3 & 4 & 3 \\
\hline 9 & 3 & 2 & 3 & 2 & & \\
\hline 10 & 3 & 3 & 3 & 4 & & 3 \\
\hline 11 & 3 & 3 & 3 & 4 & 3 & \\
\hline 12 & & & 4 & & 3 & \\
\hline 13 & 3 & & 2 & & 2 & \\
\hline 14 & 3 & 2 & & & 1 & \\
\hline 15 & & 2 & 4 & & 4 & \\
\hline 16 & 3 & 3 & & & 3 & \\
\hline 17 & 3 & & 3 & 2 & 3 & 2 \\
\hline 18 & 3 & 2 & & & 4 & 4 \\
\hline 19 & & 1 & 2 & 4 & 2 & 3 \\
\hline 20 & 1 & & 3 & 3 & 4 & \\
\hline 21 & & 2 & & & 3 & \\
\hline 22 & & 3 & 2 & & 4 & 2 \\
\hline 23 & & 4 & & 3 & 3 & 3 \\
\hline 24 & & & & 3 & 3 & \\
\hline 25 & & & & & 3 & \\
\hline 26 & & & & & 3 & \\
\hline 27 & & & & & 4 & 3 \\
\hline 28 & & & & & 4 & \\
\hline 29 & & & & & 5 & \\
\hline 30 & & & & & 4 & 3 \\
\hline 31 & & & & & 3 & 4 \\
\hline 32 & & & & & & 4 \\
\hline 33 & & & & & & 4 \\
\hline Suma de combinaciones & I & 1 & 1 & 1 & I & 1 \\
\hline N. Rep en combinaciones & 15 & 14 & 18 & 16 & 29 & 17 \\
\hline Promedio de tratamientos & & & & & & 17 \\
\hline Promedio de lotes de hembras & & 3.02 & & & 2.89 & \\
\hline
\end{tabular}




\section{COMPARATIVO DE EMPADRE CONTROLADO EN CUYES}

Asimismo, en la Tabla 7 se observará el análisis de variancia del número de crías destetadas por cada hembra parida por lotes y tratamientos. Según dicho análisis, puede decirse que las crías son semejantes para $(\mathrm{p}<0.01)$. No obstante, la diferencia de crías entre el T1, que corresponde al sistema de empadre actual que emplea Agropecuaria Inka Cuy S.A.C., y el T3, de 0.38 crías a favor de este último tratamiento, constituye una cantidad importante desde el punto de vista económico. A continuación, se detallarán los datos mencionados.

Tabla 7

Análisis de Variancia del Número de Crías Destetadas por cada Hembra Parida por Lotes y Tratamientos

\begin{tabular}{|c|c|c|c|c|c|c|c|c|}
\hline F.V. & SC & GL & $\mathrm{CM}$ & FC & $\mathrm{Ft}, 05$ & SIG & $\mathrm{Ft}, 01$ & SIG \\
\hline Entre combinaciones & 4.42 & 5 & 0.88 & 1.436 & 2.290 & No & 3.170 & No \\
\hline Entre tratamientos & 3.54 & 2 & 1.77 & 2.878 & 3.070 & No & 4.790 & No \\
\hline Entre lotes & 0.40 & 1 & 0.40 & 0.652 & 3.920 & No & 6.850 & No \\
\hline Entre interacción T x L & 0.47 & 2 & 0.24 & 0.385 & 3.070 & No & 4.790 & No \\
\hline Error Experimental & 63.43 & 103 & 0.62 & & & & & \\
\hline Total & 67.85 & 108 & & & & & & \\
\hline $\mathrm{CV}=$ & 26.48 & & & & & & & \\
\hline
\end{tabular}

Ahora bien, en la Tabla 8 se observará el número de crías destetadas por cada hembra empadrada de acuerdo a lotes y tratamientos. Las cifras ofrecen 1.84 crías para las hembras empadradas con machos que descansaron en sus propias pozas de empadre (T1) vs. 2.18 crías para las hembras empadradas con machos que descansaron en pozas individuales (maternidades) (T2) y 2.21 crías para las hembras empadradas con machos que descansaron e hicieron el efecto macho en pozas individuales (maternidades) (T3). A continuación, se especificará la información mencionada. 


\section{LUIS H. ALIAGA RODRÍGUEZ}

Tabla 8

Número de Crías Destetadas por cada Hembra Empadrada por Lotes y Tratamientos

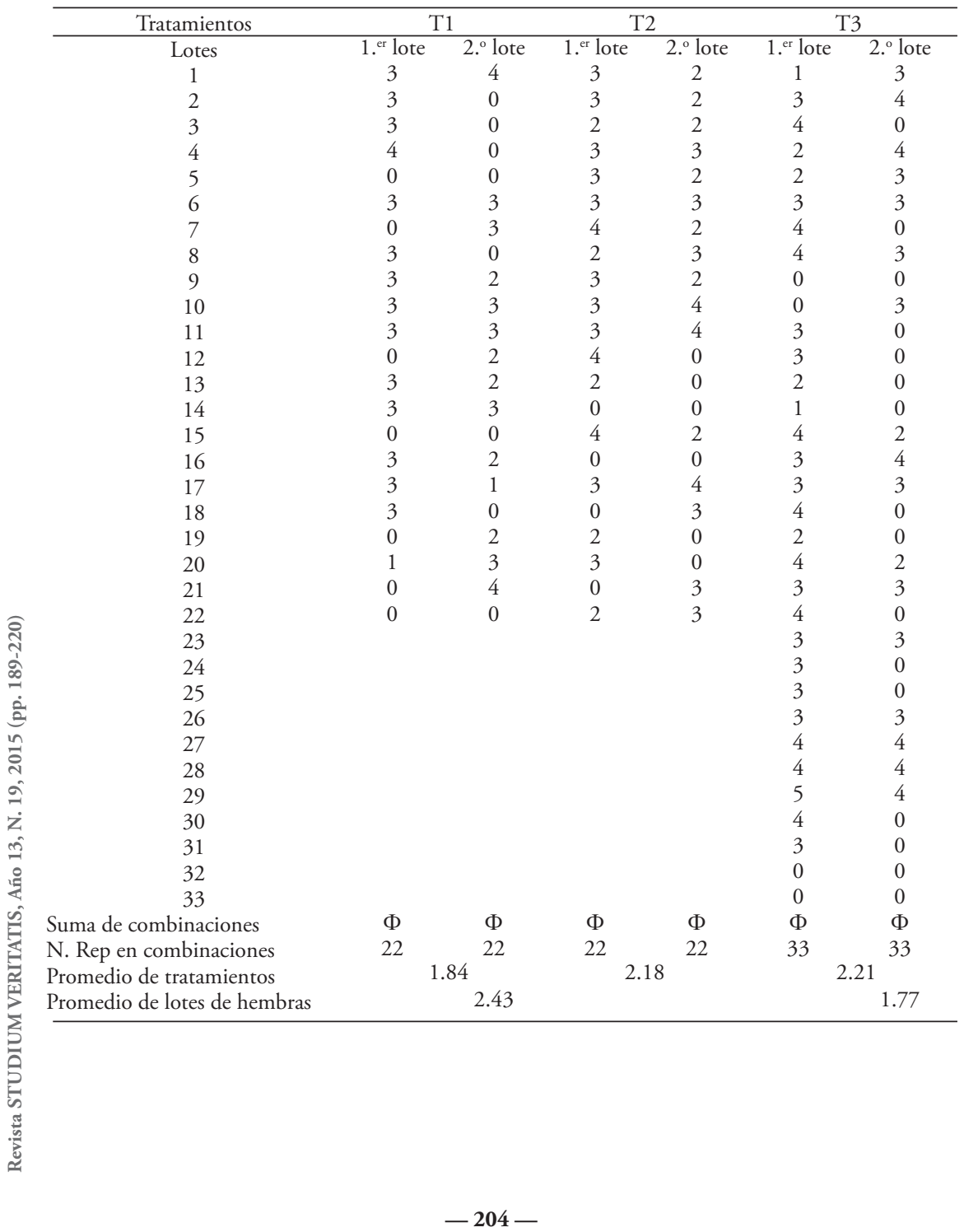




\section{COMPARATIVO DE EMPADRE CONTROLADO EN CUYES}

En la Tabla 9 se apreciará el análisis de variancia del número de crías destetadas por cada hembra empadrada por lotes y tratamientos. Como resultado puede decirse que son semejantes para $(\mathrm{p}<0.01)$. Mas la diferencia de crías entre el T1, que corresponde al sistema de empadre actual que emplea la empresa Inka Cuy S.A.C., y el T3 de 0.37 crías a favor de este último tratamiento, representa para Inka Cuy S.A.C. un capital vivo de 7,500 hembras en empadre con 4 empadres al año. Ello simboliza un incremento de 11,100 crías destetadas al año. Tal cantidad, como se verá más adelante, resultará importante en la economía de la empresa. Por lo tanto, el efecto macho, además de sincronizar el celo, permitirá una parición en un espacio de tiempo más corto, con un mayor tamaño de camada y un mayor número de crías destetadas por cada hembra empadrada.

\section{Tabla 9}

Análisis de Variancia del Número de Crías Destetadas por cada Hembra Empadrada por Lotes y Tratamientos

\begin{tabular}{lcccccccc}
\hline F.V. & SC & GL & CM & FC & Ft, 05 & SIG & Ft,01 & SIG \\
\hline Entre combinaciones & 26.28 & 5 & 5.256 & 0.036 & 2.290 & No & 3.170 & No \\
Entre tratamientos & 4.08 & 2 & 2.038 & 0.014 & 3.070 & No & 4.790 & No \\
Entre lotes & 16.89 & 1 & 16.890 & 0.114 & 3.920 & No & 6.850 & No \\
Entre interacción T x L & 5.31 & 2 & 2.657 & 0.018 & 3.070 & No & 4.790 & No \\
Error Experimental & 321.26 & 148 & 2.171 & & & & & \\
Total & 347.54 & 153 & & & & & & \\
\multicolumn{1}{c}{$\mathrm{CV}=$} & 70.24 & & & & & & & \\
\hline
\end{tabular}


En la Tabla 10, se calculará el peso total de camada destetada por cada hembra empadrada por lotes y tratamientos. Se observará, entonces, $830 \mathrm{~g}$ para el tratamiento T3; en otras palabras, $118 \mathrm{~g}$ más que el T2 y $153 \mathrm{~g}$ más que el T1. A continuación, se precisarán dichos datos.

\section{Tabla 10}

Peso Total de Camada Destetada por cada Hembra Empadrada por Lotesy Tratamientos

\begin{tabular}{|c|c|c|c|c|c|c|c|}
\hline Tratamientos & & $\mathrm{T} 1$ & & $\mathrm{~T} 2$ & & T3 & \\
\hline Lotes & 1. ${ }^{\text {er }}$ lote & 2. lote & $1{ }^{\text {er }}$ lote & $2 .^{\circ}$ lote & 1 er $^{\text {er }}$ lote & $2 .^{\circ}$ lote & \\
\hline 1 & 910 & 880 & & & & 850 & \\
\hline 2 & & & & & 590 & 1,040 & \\
\hline 3 & 140 & & & & 630 & & \\
\hline 4 & & & & & & & \\
\hline 5 & 610 & & & & 550 & 770 & \\
\hline 6 & 780 & 770 & & & & & \\
\hline 7 & 710 & 900 & 780 & & & & \\
\hline 8 & 440 & & 500 & 680 & 860 & 870 & \\
\hline 9 & & & 620 & 810 & & & \\
\hline 10 & 680 & 580 & 780 & 1,300 & & & \\
\hline 11 & 710 & 630 & 710 & & 670 & & \\
\hline 12 & & & 900 & 0 & 1,040 & & \\
\hline 13 & 700 & & 490 & 0 & & & \\
\hline 14 & 890 & 550 & & & 290 & & \\
\hline 15 & 970 & 650 & 1,300 & 870 & 670 & & \\
\hline 16 & & & & & 850 & & \\
\hline 17 & 360 & & 680 & 890 & & & \\
\hline 18 & & & & & & & \\
\hline 19 & & & & & 580 & 790 & \\
\hline 20 & & & 740 & 760 & 1,120 & & \\
\hline 21 & & & & & & & \\
\hline 22 & & & & & & & \\
\hline 23 & & & & & 660 & 840 & \\
\hline 24 & & & & & & & \\
\hline 25 & & & & & & & \\
\hline 26 & & & & & 690 & & \\
\hline 27 & & & & & 1,070 & 1,100 & \\
\hline 28 & & & & & 900 & & \\
\hline 29 & & & & & 910 & & \\
\hline 30 & & & & & 750 & 830 & \\
\hline 31 & & & & & 880 & 1,270 & \\
\hline 32 & & & & & & 1,070 & \\
\hline 33 & & & & & & 930 & \\
\hline Suma de combinaciones & $\Phi \mu \alpha$ & $\Phi \mu \alpha$ & $\Phi \mu \alpha$ & $\Phi \mu \alpha$ & $\Phi \mu \alpha$ & $\Phi \mu \alpha$ & 49,740 \\
\hline N. Rep en combinaciones & 12 & 7 & 10 & 8 & 18 & 11 & 66 \\
\hline Promedio de tratamientos & & 677 & & 12 & & 30 & 754 \\
\hline Promedio de lotes de hembras & & 728 & & & & 793 & \\
\hline
\end{tabular}




\section{COMPARATIVO DE EMPADRE CONTROLADO EN CUYES}

Los datos de la Tabla 10 se someterán a un análisis de variancia del peso total de camada destetada por cada hembra empadrada por lotes y tratamientos (ver Tabla 11). Se evidenciará que, tras dicho análisis, los pesos resultan no ser significativos. Además, el orden de mérito de estos pesos se encuentra relacionado, primero, al tamaño de camada de cada hembra parida y, después, al número de crías destetadas por cada hembra empadrada. A continuación, se mostrará la información con más detalle.

\section{Tabla 11}

Análisis de Variancia del Peso Total de Camada Destetada por cada Hembra Empadrada por Lotes y Tratamientos

\begin{tabular}{lcccccccc}
\hline \multicolumn{1}{c}{ F.V. } & SC & GL & CM & FC & Ft,05 & SIG & Ft,01 & SIG \\
\hline Entre combinaciones & 578673.76 & 5 & 115734.75 & 1.899 & 2.370 & No & 3.340 & No \\
Entre tratamientos & 312866.75 & 2 & 156433.37 & 2.566 & 3.150 & No & 4.980 & No \\
Entre lotes & 68041.31 & 1 & 68041.31 & 1.116 & 4.000 & No & 7.080 & No \\
Entre interacción T x L & 197765.70 & 2 & 98882.85 & 1.622 & 3.150 & No & 4.980 & No \\
Error Experimental & 3657253.52 & 60 & 60954.23 & & & & & \\
Total & 4235927.27 & 65 & & & & & & \\
\multicolumn{1}{c}{$\mathrm{CV}=$} & 32.76 & & & & & & & \\
\hline
\end{tabular}

La Tabla 12 simbolizará las frecuencias del efecto del descanso de machos en pozas de empadre en los partos de hembras empadradas del T1 en relación al lote 1 . Se calcularán, además, el número de días y partos, tomando en cuenta los intervalos por fechas de partos. Asimismo, se mostrarán porcentajes correspondientes a la frecuencia relativa y la frecuencia relativa acumulada. A continuación, se precisarán dichos datos. 


\section{LUIS H. ALIAGA RODRÍGUEZ}

Tabla 12

Tabla de Frecuencias del Efecto del Descanso de Machos en Pozas de Empadre en los Partos de Hembras Empadradas del T1 (Lote 1)

\begin{tabular}{cccccc}
\hline $\begin{array}{c}\text { N. de } \\
\text { días }\end{array}$ & $\begin{array}{c}\text { Intervalos por fechas } \\
\text { de partos ańo 2014 }\end{array}$ & $\begin{array}{c}\text { N. de } \\
\text { partos }\end{array}$ & $\begin{array}{c}\text { Frecuencia } \\
\text { relativa }\end{array}$ & $\begin{array}{c}\text { Frecuencia abso- } \\
\text { luta acumulada }\end{array}$ & $\begin{array}{c}\text { Frecuencia rela- } \\
\text { tiva acumulada }\end{array}$ \\
\hline 1 & 8 de junio & 0 & $0.00 \%$ & 0 & $0.00 \%$ \\
2 & 8 al 11 de junio & 0 & $0.00 \%$ & 0 & $0.00 \%$ \\
6 & 12 al 15 de junio & 4 & $26.67 \%$ & 4 & $26.67 \%$ \\
10 & 16 al 19 de junio & 1 & $6.67 \%$ & 5 & $33.33 \%$ \\
14 & 20 al 23 de junio & 1 & $6.67 \%$ & 6 & $40.00 \%$ \\
18 & 24 al 27 de junio & 3 & $20.00 \%$ & 9 & $60.00 \%$ \\
22 & 28 al 1de julio & 3 & $20.00 \%$ & 12 & $80.00 \%$ \\
26 & 2 al 5 de julio & 0 & $0.00 \%$ & 12 & $80.00 \%$ \\
30 & 6 al 9 de julio & 0 & $0.00 \%$ & 12 & $80.00 \%$ \\
34 & 10 al 13 de julio & 1 & $6.67 \%$ & 13 & $86.67 \%$ \\
38 & 14 al 17 de julio & 2 & $13.33 \%$ & 15 & $100.00 \%$ \\
\hline
\end{tabular}

En la Figura 1, se mostrará el gráfico de la curva de frecuencias del T1 en relación al lote 1 . De igual manera, se ha tomado en cuenta el descanso de machos en sus pozas de empadre de acuerdo a fechas relativas a junio y julio del 2014, año en que se realizó el estudio. A continuación, se detallarán las cifras mencionadas 


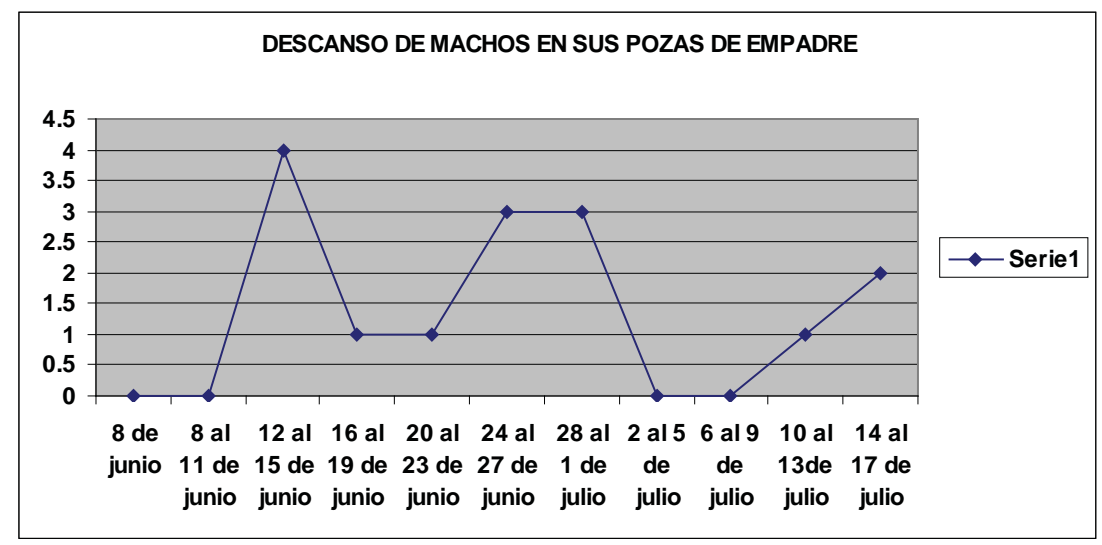

Figura 1. Gráfico de la curva de frecuencias del T1 (lote 1)

La Tabla 13 representará las frecuencias del efecto del descanso de machos en pozas individuales en los partos de hembras empadradas del T2 en relación al lote 1 . A su vez, son calculados el número de días y partos, tomando en cuenta los intervalos por fechas de partos. Igualmente, se mostrarán porcentajes correspondientes a la frecuencia relativa y la frecuencia relativa acumulada. A continuación, se detallarán los datos. 
Tabla 13

Tabla de Frecuencias del Efecto del Descanso de Machos en Pozas Individuales en los Partos de Hembras Empadradas del T2 (Lote 1)

\begin{tabular}{cccccc}
\hline $\begin{array}{c}\text { N. de } \\
\text { días }\end{array}$ & $\begin{array}{c}\text { Intervalos por fechas } \\
\text { de partos ańo 2014 }\end{array}$ & $\begin{array}{c}\text { N. de } \\
\text { partos }\end{array}$ & $\begin{array}{c}\text { Frecuencia } \\
\text { relativa }\end{array}$ & $\begin{array}{c}\text { Frecuencia } \\
\text { absoluta } \\
\text { acumulada }\end{array}$ & $\begin{array}{c}\text { Frecuencia } \\
\text { relativa } \\
\text { acumulada }\end{array}$ \\
\hline 1 & 8 de junio & 0 & $0.00 \%$ & 0 & $0.00 \%$ \\
2 & 8 al 11 de junio & 4 & $22.22 \%$ & 4 & $22.22 \%$ \\
6 & 12 al 15 de junio & 2 & $11.11 \%$ & 6 & $33.33 \%$ \\
10 & 16 al 19 de junio & 2 & $11.11 \%$ & 8 & $44.44 \%$ \\
14 & 20 al 23 de junio & 4 & $22.22 \%$ & 12 & $66.67 \%$ \\
18 & 24 al 27 de junio & 2 & $11.11 \%$ & 14 & $77.78 \%$ \\
22 & 28 al 1de julio & 0 & $0.00 \%$ & 14 & $78.78 \%$ \\
26 & 2 al 5 de julio & 1 & $5.56 \%$ & 15 & $83.83 \%$ \\
30 & 6 al 9 de julio & 1 & $5.56 \%$ & 16 & $88.89 \%$ \\
34 & 10 al 13 de julio & 2 & $11.11 \%$ & 18 & $100.00 \%$ \\
38 & 14 al 17 de julio & 0 & $0.00 \%$ & 18 & $100.00 \%$ \\
& Total & 18 & & &
\end{tabular}

La Figura 2 describirá al gráfico de la curva de frecuencias del T2 en relación al lote 1 . Asimismo, la principal variable a tomar en cuenta fue el descanso de machos en maternidades. Igualmente, los periodos descritos corresponden a junio y julio del 2014. A continuación, se ejemplificarán las cifras mencionadas. 


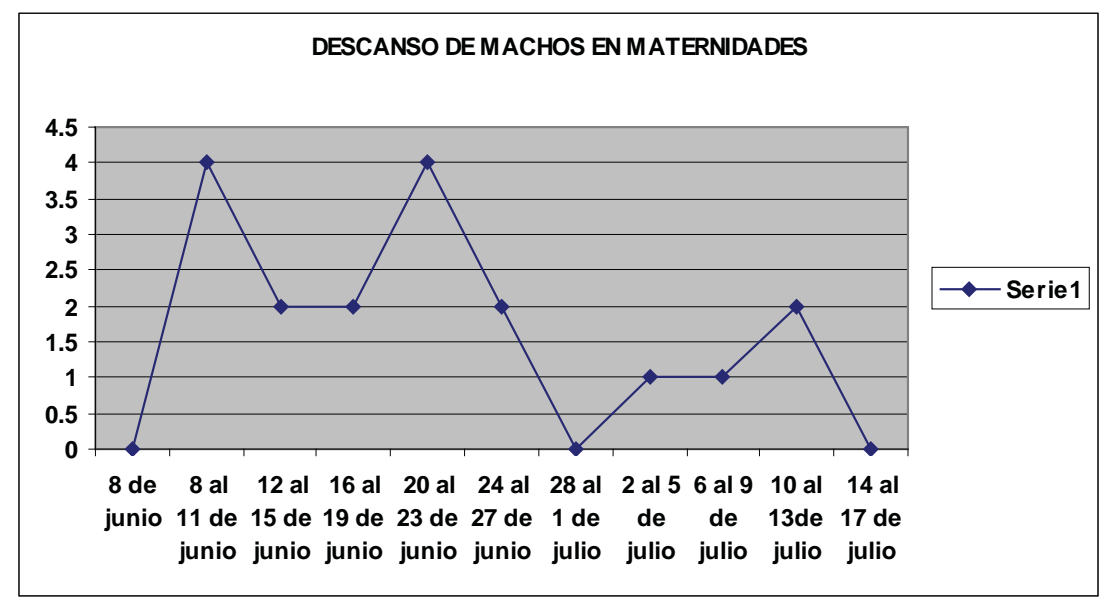

Figura 2. Gráfico de la curva de frecuencias del T2 (lote 1)

En la Tabla 14 podrán observarse las frecuencias del el efecto macho en los partos de las hembras empadradas del T3 correspondiente al lote 1. Este efecto se ha concentrado en el $79.31 \%$ de partos durante los primeros 18 días de parición, a diferencia de un $60.00 \%$ de partos en el tratamiento T1 y 77.78\% en el tratamiento T2. A continuación, se detallarán los datos. 
Tabla 14

Tabla de Frecuencias del Efecto Macho en los Partos de Hembras Empadradas del T3 (Lote 1)

\begin{tabular}{cccccc}
\hline $\begin{array}{c}\text { N. de } \\
\text { días }\end{array}$ & $\begin{array}{c}\text { Intervalos por fechas } \\
\text { de partos ańo 2014 }\end{array}$ & $\begin{array}{c}\text { N. de } \\
\text { partos }\end{array}$ & $\begin{array}{c}\text { Frecuencia } \\
\text { relativa }\end{array}$ & $\begin{array}{c}\text { Frecuencia absoluta } \\
\text { acumulada }\end{array}$ & $\begin{array}{c}\text { Frecuencia relativa } \\
\text { acumulada }\end{array}$ \\
\hline 1 & 8 de junio & 1 & $3.45 \%$ & 1 & $3.45 \%$ \\
2 & 8 al 11 de junio & 6 & $20.69 \%$ & 7 & $24.14 \%$ \\
6 & 12 al 15 de junio & 5 & $17.24 \%$ & 12 & $41.38 \%$ \\
10 & 16 al 19 de junio & 5 & $17.24 \%$ & 17 & $58.62 \%$ \\
14 & 20 al 23 de junio & 3 & $10.34 \%$ & 20 & $68.97 \%$ \\
18 & 24 al 27 de junio & 3 & $10.34 \%$ & 23 & $79.31 \%$ \\
22 & 28 al 1de julio & 3 & $10.34 \%$ & 26 & $89.66 \%$ \\
26 & 2 al 5 de julio & 3 & $10.34 \%$ & 29 & $100.00 \%$ \\
30 & 6 al 9 de julio & 0 & $0.00 \%$ & 29 & $100.00 \%$ \\
34 & 10 al 13 de julio & 0 & $0.00 \%$ & 29 & $100.00 \%$ \\
38 & 14 al 17 de julio & 0 & $0.00 \%$ & 29 & $100.00 \%$ \\
\hline
\end{tabular}

En la Figura 3 se detallará el gráfico de la curva de frecuencias del T3 en relación al lote 1 . Se ha utilizado como variable el descanso y efecto de machos en maternidades. A su vez, el resultado permitirá apreciar cómo en el T3, con efecto macho, la parición se concentra prácticamente en los primeros 18 días, debido a la sincronización del celo y preñez de las hembras en el primer ciclo estrual que, como se sabe, dura 16 días. Por otro lado, cabe mencionar que en las Figuras 2 y 1, sin efecto macho, los partos se graficarán prácticamente en dos curvas que corresponden a la preñez en los dos ciclos menstruales que coincidirán con la duración del empadre controlado (35 días). 


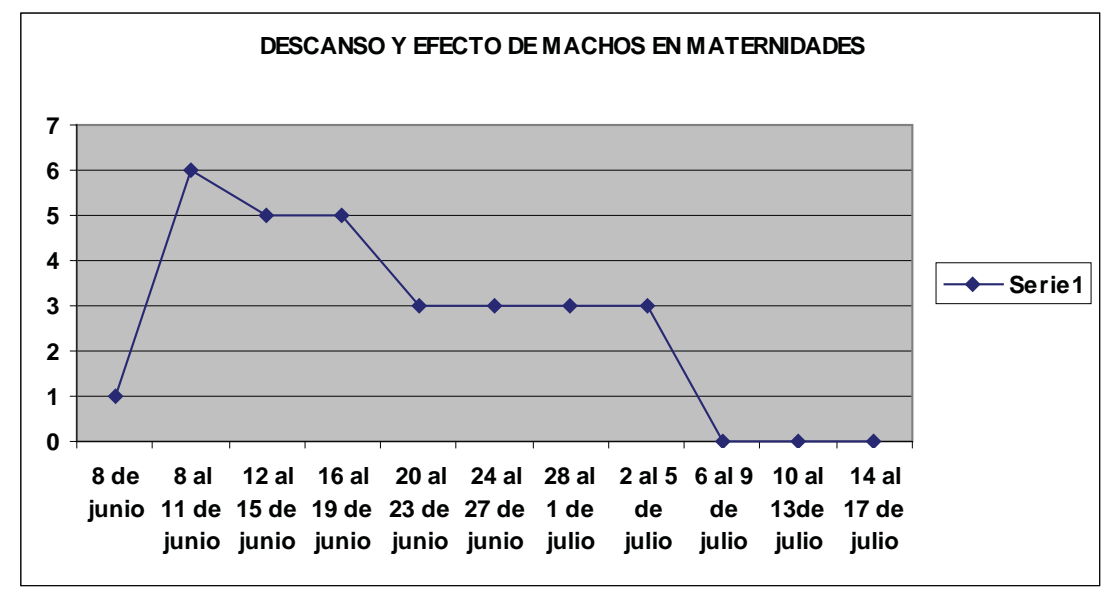

Figura 3. Gráfico de la curva de frecuencias del T3 (lote 1)

La Tabla 15 mostrará el análisis económico por tratamientos. Las variables utilizadas fueron las cantidades de hembras, números de crías, costos y valores pertinentes. Como resultado, al comparar el Valor Bruto de la Producción de Crías Destetadas, valorizadas en soles, por cada hembra empadrada, se obtiene 16.47 soles por cada hembra empadrada con machos que descansaron en sus propias pozas de empadre (T1) vs. 19.75 soles, por cada hembra empadrada con machos que descansaron en pozas individuales (maternidades) (T2) y 20.04 soles por cada hembra empadrada con machos que descansaron e hicieron el efecto macho en pozas individuales (maternidades) (T3). 


\section{LUIS H. ALIAGA RODRÍGUEZ}

\section{Tabla 15}

\section{Análisis Económico por Tratamientos}

\begin{tabular}{|c|c|c|c|}
\hline Estudio económico de los resultados & $\mathrm{T} 1$ & $\mathrm{~T} 2$ & T3 \\
\hline N. de hembras por cada poza de empadre & 11 & 11 & 11 \\
\hline N. de machos por cada poza de empadre & 1 & 1 & 1 \\
\hline \multicolumn{4}{|l|}{ Número de crías destetadas por cada hembra empadrada } \\
\hline por tratamientos según resultados del presente ensayo & 1.84 & 2.18 & 2.21 \\
\hline Número de crías destetadas por cada poza & 20.24 & 23.98 & 24.31 \\
\hline \multicolumn{4}{|l|}{ Valor de la producción de cada parto, en soles, de las crías } \\
\hline destetadas en cada poza y tratamiento considerando un & & & \\
\hline costo de 10.00 soles cada cría al destete & 202.40 & 239.80 & 243.10 \\
\hline Costo en soles de una poza de empadre & 200.00 & 200.00 & 200.00 \\
\hline \multicolumn{4}{|l|}{ Costo en soles de una poza individual para el descanso de } \\
\hline cada macho del T2 y descanso y efecto de machos del T3 & 0.00 & 20.00 & 20.00 \\
\hline Costo de 11 maternidades para cada tratamiento & 220.00 & 220.00 & 220.00 \\
\hline Costo de comederos para el concentrado & 10.00 & 10.00 & 12.00 \\
\hline $\begin{array}{l}\text { Costo total de pozas, maternidades, comederos para cada } \\
\text { tratamiento }\end{array}$ & 430.00 & 450.00 & 452.00 \\
\hline \multicolumn{4}{|l|}{ Costo de depreciación de pozas, maternidades y comederos } \\
\hline \multicolumn{4}{|l|}{ en cada empadre, considerando una vida útil de 5 años y 4} \\
\hline empadres y partos por año & 21.15 & 22.50 & 22.60 \\
\hline \multicolumn{4}{|l|}{ Valor bruto de la producción por cada poza de empadre de } \\
\hline 11 hembras al momento de destete de sus crías & 181.25 & 217.30 & 220.50 \\
\hline \multicolumn{4}{|l|}{ Valor bruto de la producción de crías destetadas, en soles, } \\
\hline por cada hembra empadrada & 16.47 & 19.75 & 20.04 \\
\hline
\end{tabular}




\section{COMPARATIVO DE EMPADRE CONTROLADO EN CUYES}

Puede observarse, entonces, que la diferencia en soles entre el T3 y el T1 (sistema actual de empadre que emplea la empresa Agropecuaria Inka Cuy S.A.C.), es de 3.57 soles a favor del T3 que, para el caso de Agropecuaria Inka Cuy S.A.C., que tiene un capital vivo de 7,500 hembras en empadre con 4 empadres al año, representa un incremento de 107,100 soles al año, cantidad importante en la economía de la empresa. Por lo tanto, el efecto macho, además de sincronizar el celo permitiendo una parición en un espacio de tiempo menor, permite un incremento en el número de crías destetadas por cada hembra empadrada y, en consecuencia, un mayor rendimiento económico por cada hembra empadrada.

\section{Discusión}

De acuerdo con los resultados, se revisará con mayor precisión a las madres que, en el primer parto de su proceso reproductivo, fueron sometidas a empadre controlado con machos que descansaron antes del empadre, en sus propias pozas de empadre, o sea, prolongando 15 días el período de empadre de 35 a 55 días (T1), vs. madres que, durante su empadre controlado de 35 días, fueron empadradas con machos que descansaron 15 días adicionales en pozas individuales (maternidades) (T2), vs. madres que durante su empadre controlado de 35 días, con machos que descansaron durante 15 días adicionales en pozas individuales (maternidades) en contacto indirecto con las hembras a empadrarse para provocar el efecto macho en la hembras (T3). Dichas comparaciones demuestran que el tamaño de camada, obtenido para el tratamiento T1, fue de 2.97; mientras que T2 consiguió 3.12 y T3, 3.29 crías. Estas, sometidas al análisis de variancia, resultan no ser significativas. A continuación, se resumirán estas cantidades en la siguiente tabla. 
Tabla 16

Resumen de los Resultados Obtenidos

\begin{tabular}{|c|c|c|c|c|}
\hline Concepto & $\mathrm{T} 1$ & $\mathrm{~T} 2$ & $\mathrm{~T} 3$ & SIG \\
\hline Peso en gramos al inicio del empadre & 857 & 847 & 892 & No \\
\hline$\%$ de hembras vacías & $29.55 \%$ & $20.45 \%$ & $19.70 \%$ & \\
\hline Tamaño de camada & 2.97 & 3.12 & 3.29 & No \\
\hline N. de crías destetadas por cada hembra parida & 2.79 & 2.82 & 3.17 & No \\
\hline N. de crías destetadas por cada hembra empadrada & 1.84 & 2.18 & 2.21 & No \\
\hline Peso total en gramos de camada al destete & 677 & 712 & 830 & No \\
\hline $\begin{array}{l}\text { Valor bruto de la producción de crías destetadas, } \\
\text { en soles, por cada hembra empadrada }\end{array}$ & 16.47 & 19.75 & 20.04 & \\
\hline
\end{tabular}

A su vez, podemos mencionar una semejanza con los resultados obtenidos por Carhuamaca y Aliaga (2005-2006) quienes, en un estudio comparativo sobre empadre controlado de 4 vs. 5 partos al ańo en la crianza สิ de cuyes, refirieron cifras de un tamaño de camada de 2.74 crías para 4 partos al año vs. 2.67 crías para 5 partos al año, sin significación estadística, cifras que son semejantes a las encontradas en este trabajo. Asimismo, Armando Borja (2009) y estudió el comportamiento de los machos en hembras sometidas a empadre controlado, considerando el número de ellos y la alternancia de los mismos demostrando que existe un efecto en los parámetros reproductivos cuando se cambia el número de ellos y cuando se alternan en el empadre. Tal situación también se corrobora en este trabajo, pues al comparar el número de crías destetadas por cada hembra empadrada en el presente estudio, se obtuvieron 1.84 crías para el tratamiento T1; 2.18 para el T2 y 2.21 para el T3 diferencias que al análisis de variancia resultan ser no significativas. 


\section{COMPARATIVO DE EMPADRE CONTROLADO EN CUYES}

Igualmente, Carhuamaca y Aliaga, en el 2013 (7), realizaron un estudio comparativo sobre empadre controlado con parición en las pozas de empadre vs. parición en maternidades. Como resultado, se obtuvieron un promedio de 2.27 crías destetadas por cada hembra empadrada para la parición en las propias pozas de empadre vs. 2.72 crías destetadas por cada hembra empadrada en parición en maternidades, sin significación estadística; cifras muy parecidas a las obtenidas en el (T1) de este trabajo. Por último, el efecto macho en cuyes, estudiado por Vicetti (1980) y Aliaga \& Braul (1983), demuestra que el contacto indirecto de los machos con las hembras previo al empadre permiten una maduración sexual y una sincronización de los celos. Dicha situación se ha comprobado en este ensayo, como pueden observarse tanto en las Tablas 13 y 14 así como en las Figuras 2 y 3 que se reportan.

\section{Conclusiones}

Luego de la muestra de resultados, se pasará a la exposición de conclusiones pertinentes al caso. A continuación, se enumerarán dichas conclusiones de acuerdo con el Sistema de Empadre Controlado en Cuyes:

- El descanso del macho en una poza individual durante 15 días, frente al descanso de los mismos en sus propias pozas de empadre, permite un incremento de 0.15 crías en el tamaño de camada y 0.34 crías destetadas por cada hembra empadrada.

- El efecto macho, en otras palabras, el contacto indirecto del macho con las hembras durante 15 días, previos al empadre, permite un mayor tamaño de camada al nacimiento de 0.17 crías por parto. Ello representa un incremento de 0.03 crías destetadas por cada hembra empadrada y un incremento de $118 \mathrm{~g}$ por peso de camada al destete. 
- El descanso del macho en una poza individual, haciendo contacto indirecto con las hembras durante 15 días $v$ s. el descanso del macho en sus propias pozas de empadre (sistema utilizado por la empresa Agropecuaria Inka Cuy S.A.C.), permite un incremento de 0.38 crías destetadas por cada hembra empadrada.

- Económicamente, el descanso del macho en una poza individual, haciendo contacto indirecto con las hembras durante 15 días (efecto macho) (T3) $v$ s. el descanso del macho en sus propias pozas de empadre (T1) (sistema actualmente utilizado por la empresa Agropecuaria Inka Cuy S.A.C.), permite un incremento en el valor bruto de la producción de 3.30 soles de crías destetadas por cada hembra empadrada.

- Si bien estos resultados al análisis de variancia no lograron significación estadística, en una explotación de cuyes el descanso del macho en una poza individual, haciendo contacto indirecto con las hembras durante 15 días (T3), resulta ser bastante económico, pues un empadre de 1,000 hembras, que es común en estas explotaciones, representa un ingreso adicional de 3,570.00 soles por cada empadre. De igual forma, tomando en cuenta 4 empadres al año, equivaldría a 14,280.00 soles anuales, cantidad que cubre de sobra la remuneración mínima vital (RMV) de un trabajador. 


\section{Referencias}

Aliaga Rodríguez, L. (1979). Producción de Cuyes. Serie: Obras de Investigación, Huancayo, Perú: Departamento de publicaciones de la Universidad Nacional del Centro del Perú.

Aliaga Rodríguez, L. (2005-2006). Estudio comparativo de empadre controlado de 4 vs 5 partos al año en la crianza de cuyes (Informe Final). Huancayo: Instituto de Investigación de la Facultad de Zootecnia. Universidad Nacional del Centro del Perú.

Aliaga Rodríguez, L., Moncayo Galliani, R., Rico Numbela, E. \& Caycedo Vallejo, A. (2009). Producción de Cuyes, Lima, Perú: Fondo Editorial de la Universidad Católica Sedes Sapientiae.

Aliaga Rodríguez, L. , \& Braúl, G. E. (1983). Efecto del macho como medio de acortar el Periodo de Parición. Huancayo, Perú: Universidad Nacional del Centro del Perú.

Borja Cueva, A. (2009). Evaluación del número de machos en el empadre controlado (Informe Final). Huancayo: Instituto de Investigación de la Facultad de Zootecnia. Universidad Nacional del Centro del Perú. 
Borja Cueva, A. (2010). Cambio de machos en el apareamiento controlado de cuyes en la G. A de Yauris (Informe Final). Huancayo: Instituto de Investigación de la Facultad de Zootecnia. Universidad Nacional del Centro del Perú.

Carhuamaca Rodriguez, O. (2013). Respuesta productiva del proceso innovado de parición de cuyes en maternidades vs parición en pozas de empadre (Informe Final). Huancayo: Instituto de Investigación de la Facultad de Zootecnia. Universidad Nacional del Centro del Perú.

Vicetti, V. R. (1980). Efecto del macho en la maduración sexual e incremento de peso del cuy hembra (Tesis, Universidad Nacional del Centro del Perú, Huancayo, Perú). 